\title{
Estimacion Del Potencial De Captura De Agua Atmosferica En Guásimas, Sonora
}

\author{
Dra. Ana Laura Bautista-Olivas \\ Dr. Fidencio Cruz Bautista
}

Universidad de Sonora, Departamento de Agricultura y Ganadería,

Hermosillo Sonora, México

Doi: 10.19044/esj.2018.v14n9p68 URL:http://dx.doi.org/10.19044/esj.2018.v14n9p68

\begin{abstract}
This paper focuses on estimating the potential of the atmospheric conditions in the locality of Guásimas Sonora for the capture of atmospheric water. To achieve this goal, an automated meteorological station was installed to record the ambient temperature, relative humidity, wind speed, and precipitation at the study site. According to the results obtained, the potential of the atmospheric conditions for the capture of water in the locality of Guásimas, in general, is medium. It presents a period of 20 to 30 days a year to capture atmospheric water with passive collectors and between 105 and 130 days during the year for the condensation of atmospheric water with active collectors.
\end{abstract}

Keywords: Fogcatcher, hygroscopy, passive collectors, atmospheric water

\section{Resumen}

El objetivo en este trabajo fue estimar el potencial que presentan las condiciones atmosféricas en la localidad de Guásimas Sonora para la captura de agua atmosférica. Para lograr este objetivo se instaló una estación meteorológica automatizada para el registro de la temperatura ambiente, humedad relativa, velocidad del viento y precipitación en el sitio de estudio. De acuerdo con los resultados obtenidos, el potencial que presentan las condiciones atmosféricas para la captura de agua en la localidad de Guásimas, en general, es medio. Pues presenta un período de 20 a 30 días al año para capturar agua atmosférica con captadores pasivos y entre 105 y 130 días durante el año para la condensación del agua atmosférica con captadores activos.

Palabras-claves: Condiciones atmosféricas, agua atmosférica 


\section{Introducción}

La falta de acceso al agua para beber, para la higiene y para la seguridad alimentaria ocasiona enormes penurias en más de mil millones de personas en el mundo (Annan, 2003). Por lo que la búsqueda de alternativas para el abastecimiento de agua debe ser una prioridad en la actualidad. Siendo la captación de niebla, de manera artificial, una práctica utilizada desde hace tiempo por algunas civilizaciones para obtener el agua (Gleason, 2005). Esta técnica se ha utilizado en aquellas regiones en donde se tienen las condiciones meteorológicas ideales para condensar la humedad atmosférica, mediante el uso de captadores pasivos (Atrapanieblas) o activos (Condensadores) que necesiten algún tipo de energía para forzar la condensación ambiental.

La captura de agua atmosférica con medios pasivos es una realidad en más de 20 países actualmente. Estos dispositivos pasivos conocidos como atrapanieblas son estructuras que se instalan con el objetivo de captar las partículas de agua que posee la niebla, permitiendo el aprovechamiento de este recurso hídrico (Schemenauer \& Cereceda, 1992; Semenzato, Falciai \& Bresci 1998). La captura de agua atmosférica pasiva ha demostrado su eficiencia en diferentes partes del mundo. Como es el caso que reportan Schemenawer, Fuenzalida y Cereceda (1988), en la comunidad de Chungungo (Chile) que se abastece con un rendimiento promedio anual de $4 \mathrm{~L} \mathrm{~m}^{-2} \mathrm{~d}^{-1}$. Marzol, (2002) menciona que en las islas Canarias los volúmenes de agua obtenida son de 4 $\mathrm{L} \mathrm{m}^{-2} \mathrm{~d}^{-1}$ en invierno y en verano y cerca de la costa hasta $5 \mathrm{~L} \mathrm{~m}^{-2} \mathrm{~d}^{-1}$.

También Estrela, Valiente, Corel y Millan (2008) reportan que en el sur de la región de Valencia España las tasas anuales de rendimiento de agua de niebla son de $7.1 \mathrm{~L} \mathrm{~m}^{-2} \mathrm{~d}^{-1}$, en tanto en el norte de esta región el rendimiento es de $4 \mathrm{~L} \mathrm{~m}^{-2} \mathrm{~d}^{-1}$. Ghassan (2009) también menciona que en las regiones altas de Arabia Saudita como en el poblado de Alsooda el rendimiento promedio es de $6.215 \mathrm{~L} \mathrm{~m}^{-2} \mathrm{~d}^{-1}$. En cambio, en las regiones más bajas como la ciudad de Abha la obtención promedio es de $3.3 \mathrm{~L} \mathrm{~m}^{-2} \mathrm{~d}^{-1}$.

Sin embargo, existen comunidades que no presentan eventos de niebla, aunque la humedad relativa sea alta (mayor 70\%). En estos casos una opción viable es la instalación de captadores activos, para que las partículas de agua puedan ser atraídas y condensadas.

La comunidad de Guásimas Sonora se caracteriza por presentar neblinas principalmente por advección. Este fenómeno se presenta cuando una corriente de aire cálido y húmedo se desplaza sobre una región más fría y tiende a un equilibrio térmico con la superficie sobre la cual se está desplazando (Petterssen, 1969). Por lo cual se considera que esta comunidad podría tener las condiciones de humedad relativa, temperatura y viento ideales para capturar el agua atmosférica de manera pasiva o activa. Pues el uso de estas técnicas se ha destacado en regiones áridas y semiáridas como es el caso de la comunidad de Guásimas Sonora. Lo que representa una alternativa útil 
para complementar el abastecimiento de agua para estas áreas remotas (AbdulWahab \& Lea 2008; Klemm et al. 2012; Schemenauer \& Cereceda 1994).

Por lo anterior el alcance de este trabajo fue estimar el potencial que presentan las condiciones atmosféricas en la localidad de Guásimas para la captura de agua atmosférica.

\section{Materiales y métodos}

El estudio se realizó en la costa de Guásimas, ubicado al sureste del municipio de Guaymas, Sonora; en las coordenadas geográficas $27^{\circ} 49^{\prime}$ $55^{\prime}$ 'N, $110^{\circ} 29^{\prime} 45^{\prime}$ ' W (Figura 1). Esta zona presenta un clima Bsh, con precipitaciones anuales de $520 \mathrm{~mm}$. Así como una temperatura promedio de $22.7{ }^{\circ} \mathrm{C}$ (Garcia, 1990).

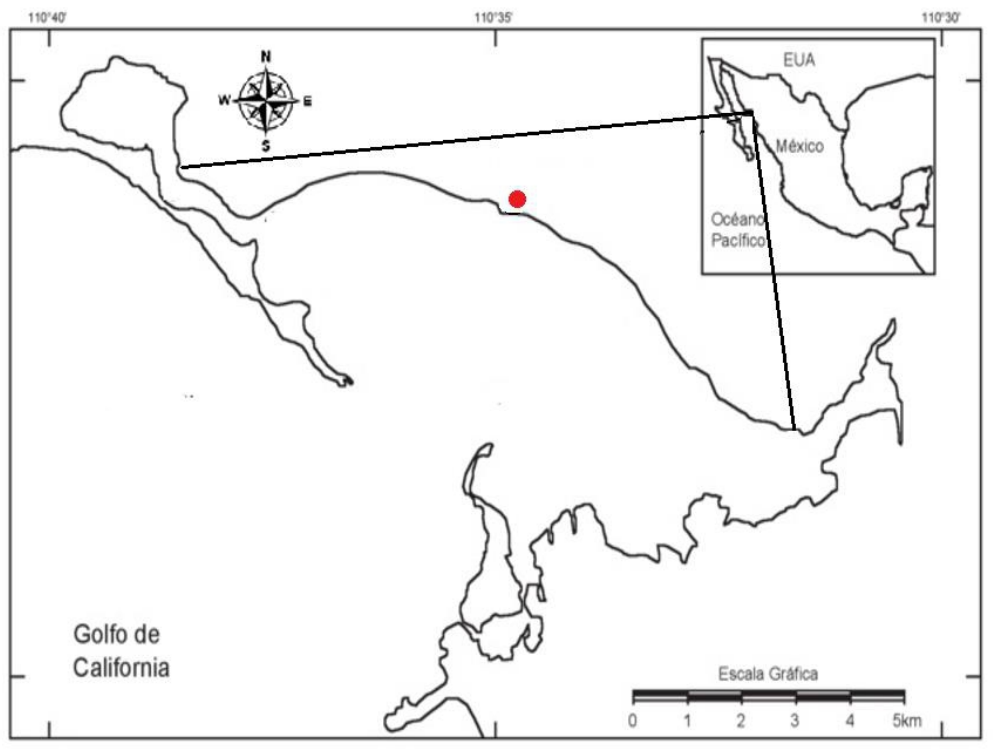

Figura 1. Localización del estudio para la captura de agua atmosférica en Guásimas, municipio de Guaymas, Sonora (Modificado de Hernández y Arrieola-lizarraga 2007).

En el sitio de estudio se instaló una estación meteorológica automatizada (EMA) modelo HOBO U-30, la cual se ubicó a una distancia de $30 \mathrm{~m}$ del mar. La EMA se programó para registrar la humedad relativa, temperatura del aire, velocidad del viento y precipitación a un intervalo de 10 minutos en un periodo de dos años (2016-2017).

El punto de rocío se calculó con la humedad relativa y temperatura obtenidos de la EMA, mediante la fórmula siguiente.

$$
\operatorname{Pr}=\sqrt[8]{\frac{H}{100}} *[112+(0.9 * T)]+(0.1 * T)-112
$$

donde: $\operatorname{Pr}=$ punto de rocío, $T=$ temperatura $\left({ }^{\circ} \mathrm{C}\right), H=$ humedad relativa 
El punto de rocío o temperatura de rocío, se refiere a la temperatura a la que empieza a condensarse el vapor de agua contenido en el aire en una superficie (Hardy, 1998).

Al considerar la premisa anterior, la captura de agua atmosférica por medios pasivos es posible cuando la diferencia entre la temperatura ambiente y el punto de rocío se encuentra en el intervalo de -1.0 a $1.8^{\circ} \mathrm{C}$, de acuerdo a lo señalado por Grillo, Entensa \& Achkienasi (2013).

Por otro lado, la captura activa se determinó en el rango de 1.9 a 5.5 ${ }^{\circ} \mathrm{C}$. Al calcular las diferencias entre la temperatura ambiente y el punto de rocío del trabajo de Bautista, Tovar, Palacios y Mancilla 2012, donde con un captador activo, obtuvieron mayor cantidad de agua a un menor costo energético. Con una diferencia de temperaturas menor de $5.5^{\circ} \mathrm{C}$.

El potencial de la captación pasiva y activa se determinó en función de los días que se tienen las condiciones ideales puede captar el agua atmosférica de acuerdo a los datos de referencia declarados de la Tabla 1.

Tabla 1. Potencial de aprovechamiento en función del número de días que se tienen las condiciones de la humedad atmosférica ideales

\begin{tabular}{ll}
\hline Días de captación pasiva & Potencial \\
\hline $50-100$ & Escaso \\
$101-200$ & Medio \\
$201-300$ & Alto \\
Mayor de 300 & Excelente \\
\hline
\end{tabular}

\section{Resultados y discusión}

De acuerdo con los resultados obtenidos en este estudio, el potencial que presentan las condiciones atmosféricas para la captura de pasiva y activa en la localidad de Guásimas, en general, es medio (Figura 2)

El número total de días del año que se tienen las condiciones atmosféricas idóneas, en el caso de la captación pasiva fueron de 20 y 29 para el año 2016 y 2017 respectivamente. Por lo cual el potencial de captura pasiva resulta escaso (Tabla 1).

En el caso de la captación activa, el total de días del año que se tienen las condiciones atmosféricas favorables fueron de 105 y 130 para el 2016 y 2017. En los meses de junio a agosto es cuando se tiene el mayor número de días favorables para la captación activa (Figura 2). 


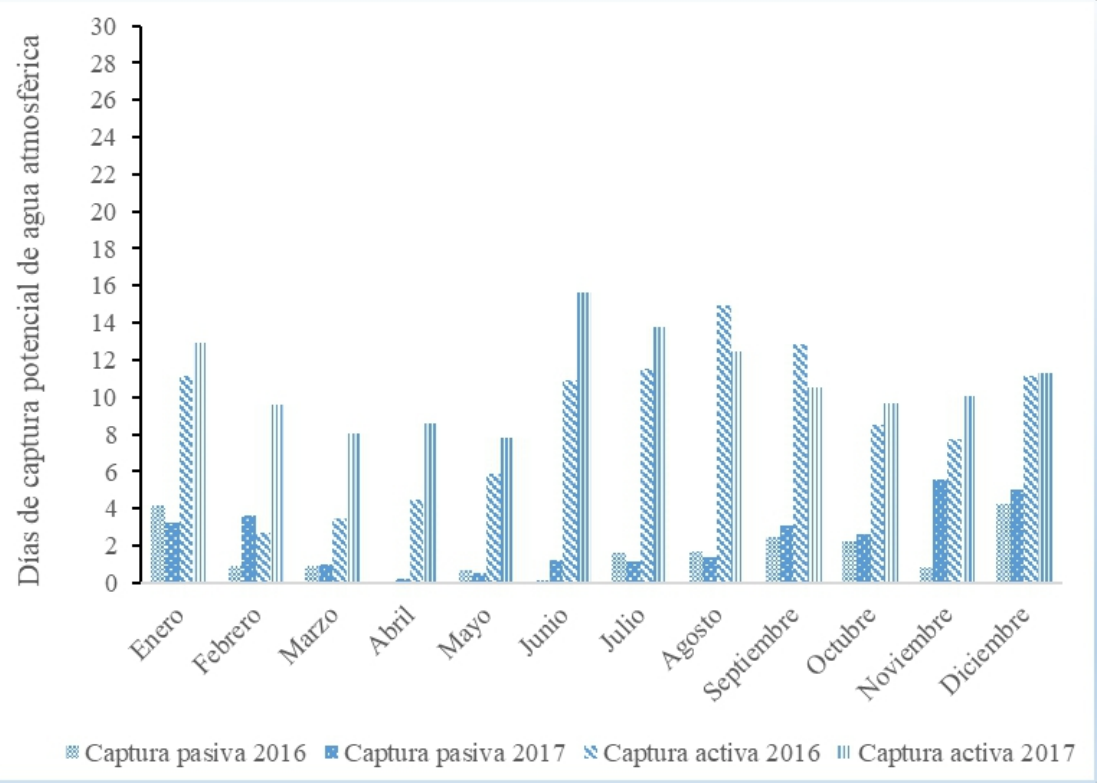

Figura 2. Días de un mes donde se presentan las condiciones ideales para la captura pasiva y activa del agua atmosférica en Guásimas, municipio de Guaymas, Sonora.

Si se considera la suma del número de días favorables para captura pasiva y activa se obtienen 125 y 159 días para el año 2016 y 2017 respectivamente. Lo cual resultaría con un potencial medio de captura del agua atmosférica, de acuerdo a los datos de referencia señalados en la Tabla 1.

Por otro lado, la tendencia de los valores de la humedad relativa y temperatura ambiente se observan en la Figura 3.

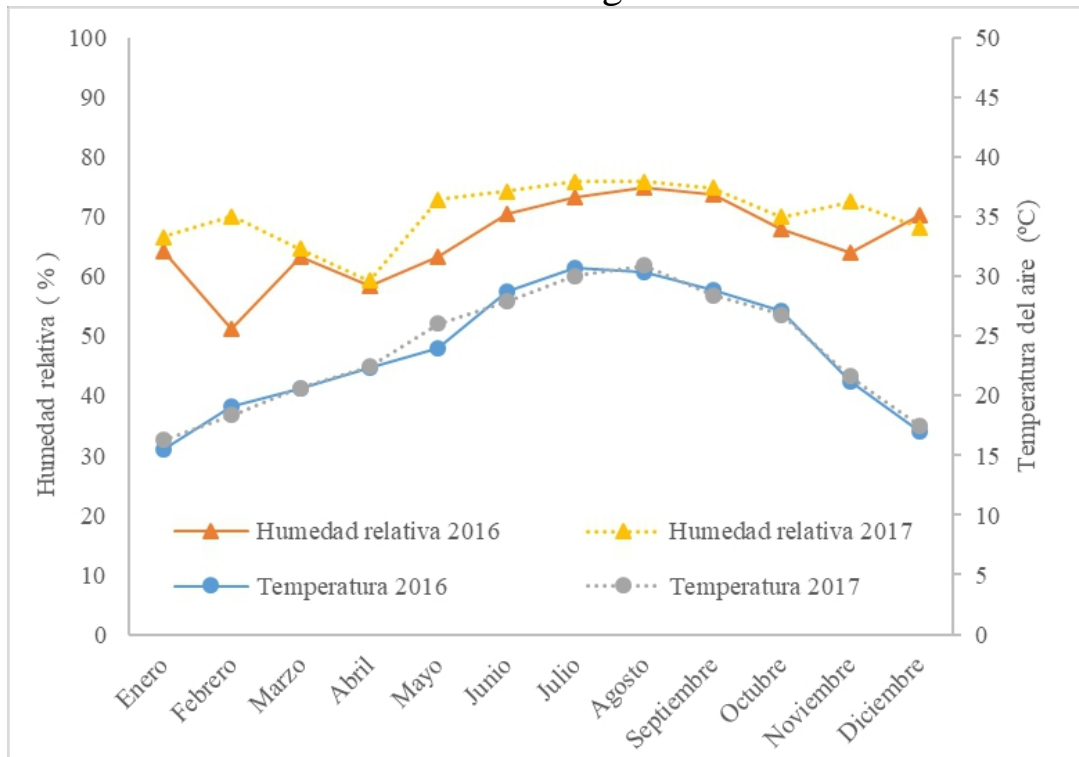

Figura 3. Tendencia de los valores de la humedad relativa y temperatura ambiente promedio durante los años 2016 y 2017 en Guásimas, municipio de Guaymas, Sonora. 
El promedio de la humedad relativa máxima fue de $75 \%$ para el mes de agosto en el 2016 y 2017. Sin embargo, en promedio la saturación de la atmósfera por arriba de $70 \%$ de humedad relativa, se presentó en cinco meses para el 2016. Mientras que para el siguiente año se presentó durante ocho meses, lo que muestra que el año 2017 fue más húmedo en un $28 \%$.

Los valores máximos de temperatura fueron de 30.72 y $30.42{ }^{\circ} \mathrm{C}$ en los meses de julio y agosto del 2016 y 2017 respectivamente. El promedio mínimo de la temperatura ambiente fue $16^{\circ} \mathrm{C}$ y se presentó en el mes de enero en ambos años.

Estos resultados son una primera aproximación del potencial que presentan las condiciones atmosféricas en la localidad de Guásimas para la captura de agua atmosférica. Estos resultados previos más estudios subsecuentes permitirá realizar un análisis de factibilidad sobre la cantidad de agua que se puede obtener y el costo energético que esto implica.

\section{Conclusiones}

De acuerdo con los resultados obtenidos en este estudio, el potencial que se tiene para la captura pasiva se considera escaso al tener un total de 20 a 30 días al año para capturar agua atmosférica.

En el caso de la captura activa se tiene un potencial medio debido a que se tienen entre 105 y 130 días durante el año para la condensación del agua atmosférica.

Si se considera la suma del número de días favorables para captura pasiva y activa se obtienen entre 125 y 150 días al año para la condensación del agua atmosférica. Lo que indica un potencial medio.

Por lo anterior, se recomienda ampliar este estudio con el fin de cuantificar la cantidad de agua que se puede captar con ambos condensadores pasivos y activos. Lo cual permitiría realizar un análisis de factibilidad para saber la conveniencia de complementar el abasto de agua a esta comunidad, por este tipo de dispositivos.

\section{Agradecimientos}

Los autores agradecen al Programa para el Desarrollo Profesional Docente (PRODEP) el apoyo económico otorgado para el desarrollo de este proyecto. También expresan su agradecimiento al Señor Pedro Higuera por las facilidades brindadas para la instalación del equipo de investigación en el sitio de estudio. Así como a Pilar Cereceda por la información proporcionada para la elaboración de la Tabla 1. 


\section{References:}

1. Abdul, W, \& Lea V (2008). Causal Factors for Nighttime Increases in Temperature Observed at a Coastal Meteorological Station. Environmental Engineering Science. 25 (6), 791-796.

2. Annan, K. (2003). Informe del Milenio. El Año Internacional del Agua Dulce. http://www.un.org/spanish/works/sustainable/freshwater.html

3. Bautista-Olivas, A. \& Tovar-Salinas, J. \& Palacios-Vélez, O. \& Mancilla-Villa, O. (2011). La humedad atmosférica como fuente opcional de agua para uso doméstico. Agrociencia 45, 293-301.

4. Estrela, M. J. \& Valiente, J.A, \& Corel, David. \& Millan, M. (2008). Fog collection in the western Mediterranean basin (Valencia region, Spain) Atmos. Res. 87, Issues 3-4, 324-337.

5. García, E. (1990). Modificaciones al sistema de clasificación climática de Köppen. Instituto de Geográfica. Universidad Autónoma de México.

6. Ghassan, A. Al-hassan (2009). Fog Water Collection Evaluation in Asir Region-Saudi Arabia. Water Resour Manage 23, 2805-2813 DOI 10.1007/s11269-009-9410-9.

7. Gleason, A. (2005). Manual de aprovechamiento de aguas pluviales en centros urbanos. Universidad de Guadalajara. Centro Universitario de Arte, Arquitectura y Diseño. Guadalajara, México.

8. Grillo, N. \& Entensa, L. Achkienasi A. (2013). La niebla en el Aeropuerto Internacional José Martí, su relación con los eventos y variables meteorológicas. Research Gate Consultado en línea en https://www.researchgate.net/publication/283568282.

9. Hardy, B.(1998). ITS-90 Formulations for vapor pressure, frostpoint temperature, dewpoint temperature, and enhancement factors in the range -100 to $100 \mathrm{C}$. International Symposium of Humidity and Moisture. London, England.

10. Hernandez y Arreola-Lizarraga (2007). Estructura de tallas y crecimiento de los cangrejos Callinectes arcuatus y $C$. bellicosus (Decapoda: Portunidae) en la laguna costera Las Guásimas, México. Rev. biol. trop 55 (1).

11. Klemm, O. \& Schemenauer, R. \& Lummerich A, \& Cereceda P, \& Marzol V, \& Corell D, \& Fessehaye G. (2012). Fog as a fresh-water resource: Overview and perspectives. Ambio 41, 221-234. doi:10.1007/s13280-012-0247-8.

12. Marzol, J. (2002). Fog water collection in a rural park in the Canary Islands (Spain). Atmos. Res. 64, 239 - 250.

13. Petterssen, S. (1969). Introduction to meteology. 3rd edition. Hardcover. 
14. Schemenauer, R., \& Cereceda, P., (1994). A proposed Standard Fog Collector for use in high-elevation regions. Journal of applied meteorology 33, 1313-1322.

15. Schemenauer, R.S., \& Cereceda, P., (1992). Water from fog-covered mountains. Waterlines 10 (4), 10- 13.

16. Schemenawer, R. \& Fuenzalida, H, \& Cereceda P. (1988). A neglected water Resource: The camanchaca of South America. Bolletin of the American Society, $69 \mathrm{pp}$.

17. Semenzato, R. \& Falciai, M. \& Bresci, E. (1998). The project Fog as a new water resource for the sustainable development of the ecosystems of the Peruvian and Chilean coastal desert. 1st Int. Conf. on Fog and Fog Collection. IDRC, (pp. 457-460) Ottawa, Canadá. 